\title{
APPROXIMATION TO REAL IRRATIONALS BY CERTAIN CLASSES OF RATIONAL FRACTIONS ${ }^{1}$
}

\author{
W. T. SCOTT
}

1. Introduction. Hurwitz proved that if $\omega$ is a real irrational number, then the inequality

$$
|\omega-p / q|<k / q^{2}
$$

is satisfied by infinitely many rational fractions $p / q$ when $k \geqq 1 / 5^{1 / 2}$, and further, that there exist irrationals everywhere dense on the real axis for which (1) is satisfied by only a finite number of fractions ${ }^{2}$ when $k<1 / 5^{1 / 2}$. He used simple continued fractions to get this result. The same result has since been obtained in two different ways by Ford. ${ }^{3}$

If $o$ denotes an odd integer and $e$ an even integer, then all irreducible fractions $p / q$ are of three classes $[o / e],[e / o]$, and $[o / o]$. It will be shown that

If $k \geqq 1$, there are infinitely many fractions of each of the three classes satisfying (1), regardless of the value of the real irrational number $\omega$.

If $k<1$, there exist irrational numbers everywhere dense on the real axis for which (1) is satisfied by only a finite number of fractions of a given one of the three classes.

The proof, like Ford's first proof of Hurwitz' theorem, will depend to a large extent on geometric properties of elliptic modular transformations.

2. Proof of the first part of the theorem. For each fraction $p / q$ construct (see Fig. 1), in the upper half-plane an $S$-circle, $S(p / q ; k)$ of radius $k / q^{2}$ and tangent to the real axis at $z=p / q$. Let $L$ be a line in the upper half-plane perpendicular to the real axis at $z=\omega$. Then (1) is satisfied by $p / q$ if and only if $L$ cuts $S(p / q ; k)$.

The group of elliptic modular transformations is the set of all transformations of the form

$$
z^{\prime}=\frac{\alpha z+\beta}{\gamma z+\delta}, \quad \alpha \delta-\beta \gamma=1,
$$

where $\alpha, \beta, \gamma, \delta$ are integers. These conformal transformations carry

1 Presented to the Society, February 24, 1940.

2 A. Hurwitz, Mathematische Annalen, vol. 39 (1891), pp. 279-285.

${ }^{8}$ L. R. Ford, Proceedings of the Edinburgh Mathematical Society, vol. 35 (1916), pp. 59-65; American Mathematical Monthly, vol. 45 (1938), pp. 586-601. 
the upper half plane into itself and the real axis into itself. Circles (including straight lines) are carried into circles. ${ }^{4}$

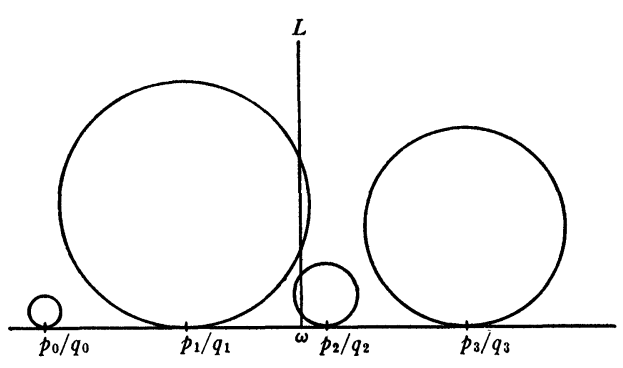

FIG. 1

Consider the subgroup

$$
G(z)=\frac{a z+2 b}{2 c z+d}, \quad a d-4 b c=1,
$$

$a, b, c, d$ integers. It is well known that a fraction $p / q$ is irreducible if and only if there exist integers $p_{0}, q_{0}$, such that $p q_{0}-q p_{0}=1$. It follows that

$$
G(p / q)=\frac{a p+2 b q}{2 c p+d q}
$$

is irreducible if $p / q$ is, since

$$
(a p+2 b q)\left(2 c p_{0}+d q_{0}\right)-(2 c p+d q)\left(a p_{0}+2 b q_{0}\right)=p q_{0}-q p_{0} .
$$

By inspection we see that $G(p / q)$ is a fraction of the same class as $p / q$. Hence, the classes $[o / e],[e / o],[o / o]$ are invariant under a transformation by any member of $G(z)$.

The set of S-circles of a given class is invariant under a transformation by any member of $G(z)$.

Because of the preceding result it will suffice to show that the set of all circles $S(p / q ; k)$ is invariant under any modular transformation.

Consider the horizontal line $y=h$, or $z-\bar{z}=2 i h$ where $h$ is a positive constant and $\bar{z}$ is the complex conjugate of $z$. When the values of $z$ and $\bar{z}$ given by the modular transformation (2) are substituted in this equation, it is found that the line is carried into $S(\alpha / \gamma ; 1 / 2 h)$. Now choose $h=1 / 2 k$ and define $S(1 / 0 ; k)$ to be the line $y=h$. The desired result is immediate.

4 A detailed account of the elliptic modular group can be found in the early chapters of L. R. Ford, Automorphic Functions, 1929, New York. 
There is a member of $G(z)$ which carries $S(p / q ; k)$ into any prescribed $S$-circle of the same class.

Let $p / q$ be a fraction of class $[o / e]$. There exist integers $p_{0}, q_{0}$, such that $p q_{0}-q p_{0}=1$; and the transformation

$$
z^{\prime}=\frac{-\left(q_{0}+n q\right) z+\left(p_{0}+n p\right)}{q z-p}
$$

carries $z=p / q$ into $z^{\prime}=\infty$. Since $p$ is odd and $q$ is even, the integer $n$ can be chosen so that this transformation is a member of $G(z)$. Similarly, there exists a member of $G(z)$ which carries the prescribed fraction of class $[o / e]$ into $z^{\prime}=\infty$. The first transformation followed by the inverse of the second transformation is a member of $G(z)$ since $G(z)$ is a group, and it is easily seen that this transformation has the desired properties.

The classes $[e / o]$ and $[o / o]$ are similarly treated, except that the $z^{\prime}=\infty$ should be replaced by $z^{\prime}=0$ and $z^{\prime}=1$.

A simple calculation shows that $S(p / q ; k)$ and $S\left(p^{\prime} / q^{\prime} ; k\right)$ are tangent if and only if

$$
k^{2}=(1 / 4)\left(p q^{\prime}-q p^{\prime}\right)^{2}
$$

If $p / q$ and $p^{\prime} / q^{\prime}$ are different fractions of the same class and $k=1$, the condition for tangency becomes

$$
p q^{\prime}-q p^{\prime}= \pm 2 \text {. }
$$

Hence, when $k=1$, two circles of the same class are either tangent or else wholly external to each other.

We note that the circles $S(1 / 2 n ; 1)$ are tangent for consecutive integral values of $n$; and the same is true for the circles $S([2 n-1] / 2 n ; 1)$. Even when $S(1 / 0 ; 1)$ is excluded these two sets of circles still have a common member; namely, $S(1 / 2 ; 1)$. These two sets of circles form a chain which extends from $-1 / 2$ to $3 / 2$, except for breaks at 0 and 1 (see Fig. 2). We apply $z^{\prime}=z+2 b$, which are members of $G(z)$, and see that there is a chain of tangent $S$-circles of class [o/e] between every pair of consecutive integral points when $k=1$.

The modular transformations

$$
z^{\prime}=-1 / z^{\prime}, \quad z^{\prime}=-z /(z-1)
$$

interchange the classes $[o / e],[e / o]$, and the classes $[o / e],[o / o]$. The chain property for class $[o / e]$ has an analogue for the two remaining classes. Only fractions of the class $[o / e]$ will be treated here, but similar treatment would yield like results for the other two classes. 
The line $L$ must cut $S(1 / 0 ; 1)$. As $L$ is traced toward $z=\omega$, an $S$-circle of a chain between two integral points must be encountered since $\omega$ is irrational. This $S$-circle is now transformed into $S(1 / 0 ; 1)$ by a suitable member of $G(z)$; and this same transformation carries $L$ into a semicircle, one of whose ends is at an irrational point. As the semicircle is traced from its intersection with $S(1 / 0 ; 1)$ toward its irrational end an $S$-circle of a chain between two integral points must be encountered. This $S$-circle is not one which has been encountered before. It is now transformed into $S(1 / 0 ; 1)$ by a suitable member of $G(z)$ and the procedure is continued. The process cannot terminate;

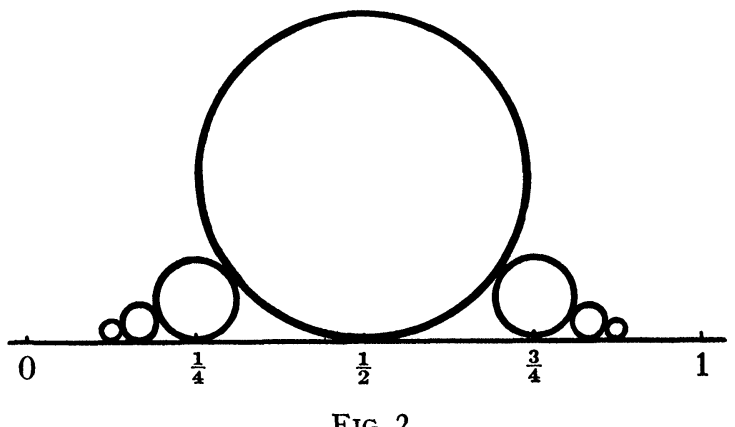

FIG. 2

for otherwise some transform of $z=\omega$ by a member of $G(z)$ would be rational, and this is not possible. This shows that $L$ cuts infinitely many $S$-circles of class $[o / e]$ when $k=1$.

3. Proof of the second part of the theorem. We shall show that there exist irrational numbers for which the inequality (1) is satisfied by only a finite number of fractions of class $[o / o]$ when $k<1$. The geometric properties used to show this are preserved by the modular transformations and hence this result will be valid for the other two classes. The proof will then be complete since the transforms of any point on the real axis by $G(z)$ are everywhere dense on the real axis.

Let $K_{n}$ be a semicircle with center at $z=-n$, where $n$ is a positive integer, and tangent to $S\left(-1 / 1 ; k_{n}\right)$ and $S\left(1 / 1 ; k_{n}\right)$. Because of the tangency conditions the radius of $K_{n}$ is

$$
r_{n}=\left((n-1)^{2}+k_{n}^{2}\right)^{1 / 2}+k_{n}, \quad r_{n}=\left((n+1)^{2}+k_{n}^{2}\right)^{1 / 2}-k_{n} .
$$

On solving these equations for $r_{n}$ and $k_{n}$ we get

$$
r_{n}=\left(n^{2}+1\right)^{1 / 2}, \quad k_{n}=n\left(n^{2}+1\right)^{-1 / 2} .
$$

The two points at which $K_{n}$ cuts the real axis are $-n \pm\left(n^{2}+1\right)^{1 / 2}$, and 
these are the roots of $z^{2}+2 n z-1=0$. If the left member is regarded as a quadratic form in $z$ and 1 , Pell's equation is

$$
t^{2}-\left(n^{2}+1\right) u^{2}=1 \text {. }
$$

A solution is obviously $t=2 n^{2}+1, u=2 n$, and this serves to determine the modular transformation

$$
T_{n}(z)=\frac{z+2 n}{2 n z+\left(4 n^{2}+1\right)}
$$

whose fixed points are the ends of the semicircle ${ }^{5} K_{n}$. But any circle passing through the fixed points of a modular transformation is a fixed circle for the transformation. Then any circle through the ends of $K_{n}$ is a fixed circle for $T_{n}(z)$. We note also that $T_{n}(z)$ is a member of $G(z)$.

The transformation $T_{n}(z)$ carries $S\left(-[2 n+1] / 1 ; k_{n}\right)$ into $S\left(1 /[2 n-1] ; k_{n}\right)$; and the arc of $K_{n}$ lying between its points of tangency with these circles is carried into an arc of $K_{n}$ which adjoins the original arc at its point of tangency with $S\left(1 /[2 n-1] ; k_{n}\right)$. Repeated application of $T_{n}(z)$ and its inverse will cover all of $K_{n}$, its end points excluded, by transforms of this arc. If no $S$-circles of class $[o / o]$ intersect the arc of $K_{n}$ between $S\left(-[2 n+1] / 1 ; k_{n}\right)$ and $S\left(1 /[2 n-1] ; k_{r}\right)$, it will follow that $K_{n}$ is tangent to infinitely many $S$-circles of class $[0 / 0]$ and intersects none.

It is clear that no $S$-circle of class $[o / o]$ can cut $K_{n}$ in the arc between $S\left(-[2 n+1] / 1 ; k_{n}\right)$ and $S\left(1 / 1 ; k_{n}\right)$ since the $S$-circles are external to each other and have maximum diameter 2 . The condition that no $S$-circle of class $[0 / 0]$ intersect $K_{n}$ in the arc between $S\left(1 / 1 ; k_{n}\right)$ and $S\left(1 /[2 n-1] ; k_{n}\right)$ is found to be

$$
p^{2}+2 n p q-q^{2} \geqq 2 n,
$$

where $p$ and $q$ are odd positive integers, $q<2 n$. This can be written in the form

$$
2 n+(2 n q+p+1)(p-1)+(2 n-1-q)(q-1) \geqq 2 n,
$$

which obviously holds, since no terms are negative.

Consider now any value of $k<k_{n}$. The $S$-circles for $k$ are smaller than those for $k_{n}$ and none of class $[o / o]$ touch $K_{n}$. Semicircles $C^{\prime}$, $C^{\prime \prime}$, one inside and the other outside $K_{n}$, which pass through the ends of $K_{n}$ and intersect no $S$-circles of class [o/o] can be constructed (see

5 A discussion of Pell's equation can be found in G. B. Mathews, Theory of Numbers, part I, Cambridge, 1892. 
Fig. 3) since all such semicircles are invariant under $T_{n}(z)$. There are no $S$-circles of class $[0 / o]$ in the region between $C^{\prime}$ and $C^{\prime \prime}$. If $L$ is a

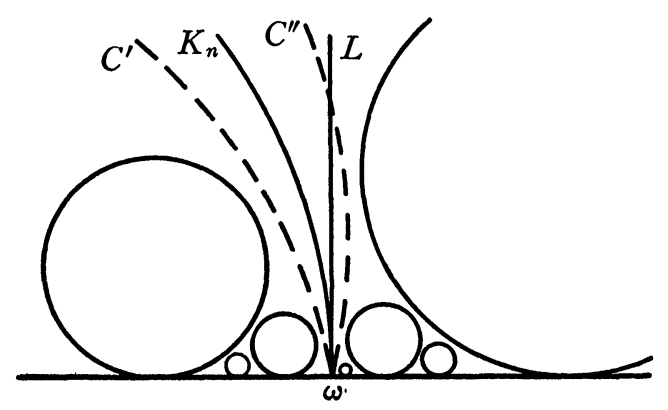

FIG. 3

line terminating at one of the end points of $K_{n}$, then near the real axis it lies in the region between $C^{\prime}$ and $C^{\prime \prime}$. Hence $L$ cuts at most a finite number of $S$-circles of class $[0 / 0]$.

Thus, for any $k<1$, we need only to choose a positive integer $n$ such that $k<n\left(n^{2}+1\right)^{-1 / 2}$. The result obtained above shows that the irrationals $-n \pm\left(n^{2}+1\right)^{1 / 2}$ have only a finite number of fractions of class $[o / o]$ satisfying (1) for this value of $k$. This completes the proof of the theorem.

Armour Institute of Technology 